\title{
Kernos
}

Revue internationale et pluridisciplinaire de religion grecque antique

$2 \mid 1989$

Varia

\section{Platon et la dimension religieuse de la procréation}

\section{André Motte}

URL : http://journals.openedition.org/kernos/246

DOI : 10.4000/kernos.246

ISSN : 2034-7871

\section{Éditeur}

Centre international d'étude de la religion grecque antique

Édition imprimée

Date de publication : 1 janvier 1989

Pagination : 157-173

ISSN : 0776-3824

Référence électronique

André Motte, "Platon et la dimension religieuse de la procréation », Kernos [En ligne], 2 | 1989, mis en ligne le 02 mars 2011, consulté le 20 avril 2019. URL : http://journals.openedition.org/kernos/246 ; DOI : 10.4000/kernos.246 
Kernos, 2 (1989), p. 157-173.

\section{PLATON ET LA DIMENSION RELIGIEUSE DE LA PROCRÉATION}

Suivre à la trace le thème de la procréation amène à détacher de la trame serrée que forme la philosophie platonicienne des fils nombreux et d'importance. La politique, l'éthique et le droit sont concernés au premier chef, mais l'anthropologie, la physique et la métaphysique y sont également engagées. Si ce thème, à notre connaissance, n'a jamais fait l'objet d'une enquête d'ensemble qui en examinerait conjointement toutes les facettes, tant s'en faut cependant qu'il soit resté jusqu'ici inexploré. On peut citer plusieurs études qui l'ont récemment abordé, de biais ou de face, contribuant, chacune de leur point de vue, à une mise en perspective des positions souvent originales défendues par le philosophe ${ }^{1}$. Ce sont les aspects religieux du thème, abondants et complexes, que nous avons choisi pour notre part d'examiner. Nous en avons regroupé, dans chaque œuvre concernée, les éléments dispersés en tenant compte des sujets traités et en nous efforçant aussi de distinguer les principaux types de discours dont ils relèvent.

\section{Le discours prescriptif}

La République : une conception purement politique de la procréation et du sacré qu'elle comporte

Dans l'espèce d'épure que dessine ce dialogue, la procréation n'est envisagée que sous le rapport, à la fois quantitatif et qualitatif, de la perpétuation de la cité : comment assurer l'excellence du troupeau (V, 459d-e). Seule est d'ailleurs concernée la classe des gardiens. Les éléments religieux qui accompagnent l'énoncé des directives s'inscrivent dans cette préoccupation

1 Platon est très souvent convoqué par Michel FouCAULT dans le deuxième volume de son Histoire de la sexualité. L'usage des plaisirs, Paris, Gallimard, 1984; nous aurons à nous démarquer de certaines thèses que défend cẹt ouvrage au demeurant très brillant. Une remarquable étude critique en a été faite par Marcel PIÉRART, Michel Foucault et la morale sexuelle des Anciens, in FZPhTh, 33 (1986), p. 23-43. Nous avons mis à profit également plusieurs articles récemment publiés par un de nos anciens élèves et collaborateurs : Jacques BELs, La procréation de Platon à Héraclite. Note sur le refus d'une influence, in Rev. des Sc. phil. et rel., 69 (1985), p. 400-408; Procréation et philosophie. Notes sur la conception de la procréation dans la philosophie de l'Antiquité, in $R P h L, 84$ (1986), p. 445-459; voir aussi La procréation dans la pensée d'Héraclite, in Rhilos , 1 (1986), p. 31-37. 
politique; ils leur apportent la caution supérieure dont elles ont besoin pour être acceptées.

On peut s'attendre à ce que les mesures décrétées soient d'autant plus sévères que, ne pouvant égaler la perfection de la génération divine ( $\tau$ ò $\theta$ eîov

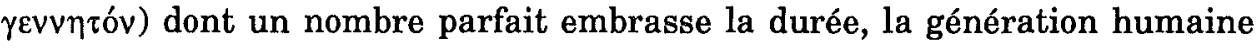
porte en elle le principe de sa dissolution au bout d'un temps (VIII, 546a-e).

La communauté des biens, des femmes et des enfants, réputée la partie la plus excellente de la constitution, abolit les familles afin de mieux réaliser, au sein de la classe, l'accord unanime des intérêts et des sentiments ${ }^{2}$. Le mantien d'une institution matrimoniale $(\gamma \alpha \dot{\alpha} \mu \varsigma)$ pour présider aux accouplements n'en est que plus significatif. Sous la poussée de l'instinct érotique

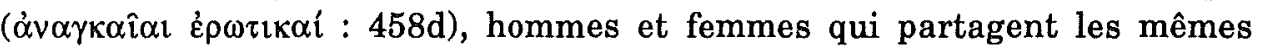
occupations seront en effet entraînés à s'unir. Mais, s'agissant de procréer pour la cité des enfants qui soient «les meilleurs et les plus utiles», il serait

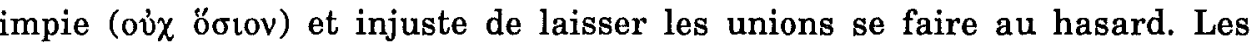
magistrats y veilleront donc avec le plus grand soin, l'objectif, présenté

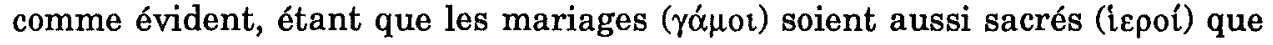
possible. Le législateur précise aussitôt ce qu'il convient d'entendre par là :

Que soient tenus pour sacrés ceux qui sont les plus avantageux ${ }^{3}$.

Et comment le seront-ils ? On s'arrangera pour masquer sous d'ingénieux tirages au sort les procédés mis en ouvre afin que s'accouplent le plus souvent possible les sujets d'élite. Le chiffre de la population devant rester stable, la sagesse des magistrats aura à déterminer la fréquence des unions. Celles-ci auront lieu au cours de fêtes ( fices et des prières que réciteront à chaque fois les prêtres, les prêtresses et toute la cité afin que naissent de ces hommes d'élite des enfants meilleurs et plus utiles encore; des poètes seront en outre chargés de composer des hymnes appropriés aux célébrations.

Très solennelles, comme on le voit, ces cérémonies sont le cadre exclusif dans lequel il est permis aux hommes et aux femmes gardiens de s'accoupler; s'il devait arriver que naisse un enfant dont les parents n'auraient pas été unis par un magistrat, il ne serait qu'un bâtard, dépourvu

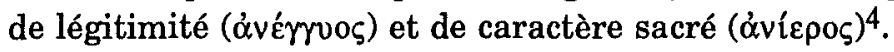

2 Tous les textes platoniciens relatifs au mariage et à la procréation que nous

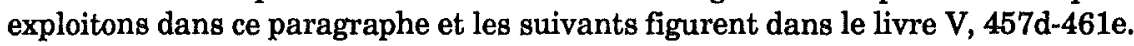

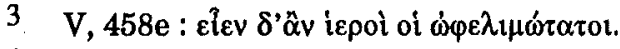

4 C'est le seul emploi de cet antonyme dans toute l'œuvre de Platon. Sur le vocabulaire qui exprime la notion de sacré chez cet auteur, voir notre étude : L'expression $d u$ sacré chez Platon, in REG, 103 (1989), sous presse. Notons encore cette autre 
L'âge pour procréer sera lui-même limité à la période, différente pour l'homme et pour la femme, où le corps et l'esprit sont en leur pleine force. On décrétera donc que ce serait une faute contre la piété et la justice (oü $\tau \varepsilon$ öotov,

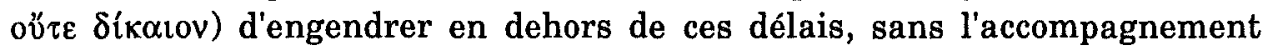
du rituel prévu; un tel enfant serait considéré comme l'œuvre des ténèbres et d'une funeste intempérance.

Passé l'âge officiel de la procréation, hommes et femmes seront libres de s'unir à qui ils voudront, hormis aux membres ascendants et descendants de leur lignée. Mais tout devra être mis en cuvre pour éviter que naisse un enfant. Si les précautions prises venaient à échouer, il doit être entendu que la cité ne nourrira pas ce rejeton. Pareillement d'ailleurs, elle se débarrassera des enfants nés dans les conditions légitimes, mais qui seraient difformes ou issus de citoyens d'une qualité inférieure; elle les dissimulera dans un endroit que l'on doit taire ( $\dot{\alpha} \pi$ ó $\rho \eta \tau o \zeta)$ et soustraire aux regards.

Quant aux enfants qui répondent à tous les critères de cet eugénisme, ils seront administrés par un comité mixte qui les confiera à des gouvernantes, dans un quartier séparé de la ville, et veillera à ce qu'ils soient nourris au sein, soit par leurs propres mères, - mais il faudra éviter qu'elles ne reconnaissent leur progéniture, - soit par des nourrices.

Dans cette grande et harmonieuse famille que formera ainsi la race des gardiens, les plus jeunes donneront à tous leurs géniteurs anonymes le nom de parents et leur témoigneront le respect, la sollicitude et la soumission

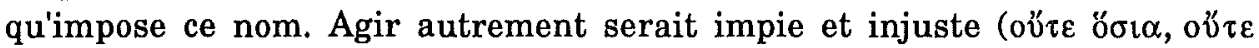

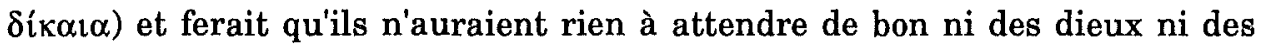
hommes (463d).

Dans les éléments religieux que met en œuvre cette étonnante législation, les lecteurs athéniens pouvaient assurément reconnaitre certaines coutumes et notions qui leur étaient familières. Le mariage revêtait bien chez eux un caractère sacré; il était censé reproduire, d'une certaine manière, le mariage des dieux dont la cérémonie, répétée annuellement, avait ce caractère rituel, purement ponctuel et efficace que l'on retrouve dans la République à propos de l'union des gardiens. A Athènes, précisément, on se mariait de préférence au mois de Gamélion durant lequel était célébrée la théogamie de Zeus et d'Héra, honorés pour la circonstance comme «maítres

référence religieuse (461e) : la loi permettra au besoin l'union de frères et de sœurs si le tirage au sort le décide et si la Pythie le confirme. 


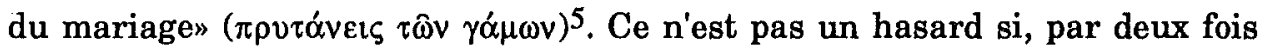
(459a, 462a), le nom de Zeus est invoqué par Socrate dans ce passage. Le mariage divin était ainsi conçu comme le garant des mariages humains; implorer les dieux, en cette circonstance, pour s'assurer une heureuse descendance relevait de la piété la plus réflexe. La fête athénienne des Anthestéries au cours de laquelle était mimé le mariage de Dionysos avec la reine de la cité, femme de l'archonte-roi, rendait aussi manifeste à tous la signification plus proprement politique de certains mariages sacrés.

En les auréolant d'un prestige sacré lié à de vieilles traditions, Platon avait chance de mieux faire admettre les institutions curieusement contraignantes qu'il proposait et dont il savait qu'elles devaient heurter ses lecteurs. Dans "la cité des heureux" qu'il imagine règne un utilitarisme strict que déroule une froide logique. Les règles régissant la procréation ne prennent en compte que l'intérêt supérieur de la cité : ni l'enfant, ni les parents, ni le couple éphémère qu'ils forment ne sont considérés en eux-mêmes. Et cet ordre politique se confond avec l'ordre religieux (őoıov) : est réputé sacré et sanctionné par les dieux ce qui est censé concourir au bien de la cité. La génération humaine doit prendre résolument pour modèle la parfaite rationalité de la génération divine.

\section{Les Lois : une vision plus éthique et plus religieuse}

Le législateur, cette fois, s'adresse à l'ensemble de la cité et renonce à son utopie d'une communauté des femmes et des enfants, restituant du même coup à la famille son rôle traditionnel. A l'encontre du droit classique, Platon n'en maintient pas moins la conception d'un mariage finalisé par la procréation et subordonné à la constitution sociale de la cité6. La préoccupation fondamentale reste la même : faire en sorte que soient engendrés pour la cité les enfants les meilleurs sous tous rapports (VI, 783d). Mais on verra que ce principe ménage cette fois d'autres perspectives.

5 Schol. à ARISTOPHANe, Thesmoph., 973-976 Dübner. Selon Olympiodore (Com. in Arist. Meteora, I, 6), les Athéniens choisissaient cette période de l'année pour se marier, parce qu'ils estimaient raisonnable de «semer» au moment où la terre est arrosée, ce temps étant propice à la naissance des fruits. Il y avait à Athènes une statue de Gè suppliant Zeus de faire pleuvoir sur elle : PAUS., I, 24, 3. Sur les théogamies attiques, voir l'article de F. SALVIAT, in $B C H, 88$ (1964), p. 647-654. Sur les thèmes mythiques du mariage sacré, voir notre ouvrage Prairies et jardins de la Grèce antique. De la religion à la philosophie, Bruxelles, 1973, p. 214-232.

6 Voir à ce sujet L. Gernet, in Platon, Les Lois, Paris, Belles Lettres, t. 1, Intr., p. CLXIV sq. 
Le chiffre global de la population, déterminé avec précision sur base de

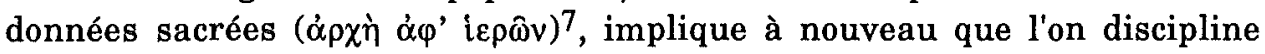
soigneusement la fécondité. La première préoccupation du législateur concernera donc ce point de départ des naissances dans la cité qu'est l'organisation des mariages (IV, 721a). Il décrétera pour chaque citoyen l'obligation légale de procréer allant de pair avec celle de se marier (VI, 774a).

Les mesures d'accompagnement sont exposées de façon beaucoup plus complète et plus concrète que dans la République. Platon se montre constamment soucieux d'imprégner sa législation d'un caractère sacré qui la rend familière et vise parfois à émouvoir. La dimension religieuse est singulièrement enrichie par diverses considérations.

L'attention des colons est attirée sur le fait que certains lieux, en raison de particularités géographiques, mais aussi de la présence d'un souffle divin et de démons, sont plus aptes que d'autres à engendrer des hommes bons (VI, 747d-e). Quant aux unions matrimoniales, il convient de les préparer de loin en offrant aux jeunes l'occasion de se rencontrer et de se connaître à la faveur de fêtes où l'on se concilie la faveur des dieux (VI, 771d-e). Chacun aura à cœur cependant de rechercher le mariage qui sert la cité et non celui qui lui serait le plus agréable.

Pour la cérémonie des fiançailles ( $\left.\pi \rho \tau \tau \ell \lambda i^{\alpha} \alpha \gamma \alpha \dot{\alpha} \mu \omega v\right)$ et les autres rituels

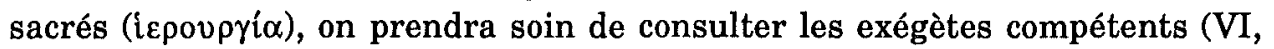
775a). Ces fêtes, accompagnées de sacrifices, seront l'occasion de rappeler aux époux leurs devoirs. Ceux qui recevraient ou donneraient en dot un montant dépassant la norme prescrite seraient taxés d'une somme qui sera consacrée à Héra et à Zeus (VI, 774d) ${ }^{8}$. Au banquet de noces, qui sera modeste, on veillera tout spécialement à ce que les époux restent sobres. L'important changement que représente le mariage dans leur vie ainsi que la perspective de l'enfant à concevoir imposent en effet qu'ils soient en pleine possession de leur esprit :

Car on ne sait guère quelle nuit ou quel jour, avec l'aide divine ( $\mu \varepsilon \tau \grave{\alpha}$ $\theta \varepsilon 0 \hat{)})$, le feront naître (VI, 775c).

7 VI, 771a; ce chiffre est de cinq mille quarante habitants. Le nombre d'enfants considéré par la loi comme suffisant pour chaque foyer est d'au moins un garçon et une fille (XI, 930d). La remarque est incidente.

8 Une amende annuelle dont bénéficie aussi Héra est à verser par celui qui ne se marierait pas à l'âge fixé (VI, 774a-b). 
Toute la vie et, plus encore, durant tout le temps que l'on procrée, il faut se garder de ce qui est malsain ou relève de la démesure et de l'injustice. Mais c'est surtout ce jour et cette nuit-là que l'on s'abstiendra de pareilles fautes :

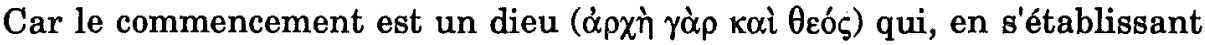
chez les hommes, sauve toutes choses, si chacun de ses dévots lui rend l'honneur convenable (VI, 775c, trad. des Places).

Dans cette longue exhortation à la sobriété et à la vertu, le ton s'est fait pressant et le philosophe veut toucher les cceurs, soulignant avec insistance le mystère sacré qui plane sur la conception.

Les instructions qui suivent sont également marquées d'une note existentielle. Elles disent que les jeunes mariés devront habiter une maison en propre où naîtront et grandiront leurs petits. Ils quitteront donc leurs parents : c'est qu'une cohabitation prolongée empêcherait ce regret bénéfique qui naît d'une séparation et risquerait de compromettre l'affection qu'ils ont pour eux. Mais ils leur rendront assurément visite et les recevront, tandis qu'eux-mêmes seront préoccupés de

transmettre d'une génération à l'autre le flambeau de la vie, ne cessant ainsi de servir les dieux conformément aux lois ${ }^{9}$.

Pour bien réussir une entreprise, il faut y appliquer son esprit. Que l'époux soit donc attentif à sa femme et à la procréation ( $\pi \alpha$ เoonoría), et que la femme fasse de même, surtout durant le temps qui précède la venue d'enfants. Un collège de femmes veillera d'ailleurs sur les jeunes foyers. $\mathrm{Au}$ cours de la réunion quotidienne qu'elles tiendront dans le temple d'Ilithye, elles se signaleront mutuellement les ménages en âge de procréer où l'on faillit à son devoir. Si les avertissements et les menaces se révèlent insuffisants, il y aura dénonciation publique et, en cas de condamnation, interdiction de prendre part aux fêtes religieuses qui célèbrent les mariages et les naissances. Cette surveillance durera dix ans et les unions qui, au terme de ce délai, seraient restées infécondes seront normalement dissoutes (VI, 783c-784d).

Cette dernière précision confirme, si besoin en était, combien le mariage reste, aux yeux de Platon, ordonné à la procréation. Stérile, il serait un

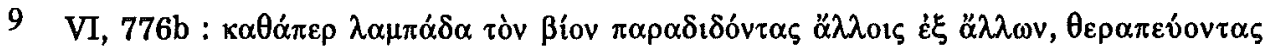

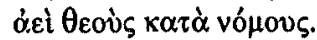


contre-exemple, et peut-être considère-t-il aussi qu'une telle situation contreviendrait au caractère sacré de l'institution, l'infécondité pouvant être le signe de la défaveur des dieux. Au demeurant, les liens familiaux font l'objet dans les Lois d'une sollicitude toute particulière et se voient sans cesse valorisés par une référence religieuse.

Procréation, soins accordés aux enfants, honneurs dus aux parents, culte des ancêtres et des dieux sont présentés comme étroitement solidaires et envisagés dans une continuité profonde. La famille possède ses propres sanctuaires où les enfants sont inscrits à leur naissance (VI, 785a). Elle est

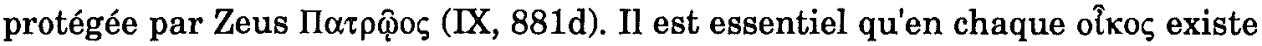
un propagateur de la race qui maintienne le foyer et soit en même temps le

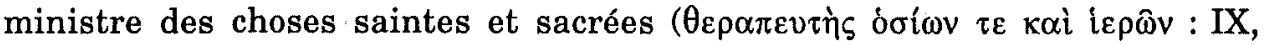
878a). Celui qui vénérera $(\sigma \varepsilon ́ \beta \varepsilon \sigma \theta \alpha \mathrm{l})$ les membres de sa parenté, - ceux qui ont même sang et mêmes dieux familiaux, - pourra escompter la bienveillance des dieux de la naissance ( $\gamma \varepsilon v \varepsilon ́ \theta \lambda$ ror $\theta \varepsilon o i ́$ ) pour la procréation de ses propres enfants $(V, 729 \mathrm{c})$. Plus particulièrement, le culte dû aux parents inspire à l'auteur des Lois des pages qui comptent parmi les plus émouvantes. C'est en les honorant, et ces exigences sont longuement détaillées, que chacun pourra obtenir des dieux la part qu'il attend et "passer le meilleur de sa vie dans de douces espérances» (VI, 717b-719a). Nos parents ne sont-ils pas, parmi nous, commes des statues, non point inertes comme le sont celles des dieux, mais vivantes, et combien efficaces pour attirer sur nous les bénédictions divines $(\mathrm{XI}, 930 \mathrm{e}-932 \mathrm{a})^{10}$ ?

La procréation crée ainsi, entre parents et enfants, des liens de réciprocité dont Platon tient beaucoup à souligner le caractère sacré. Dans la famille, la relation verticale l'intéresse manifestement davantage que la relation horizontale. On constate cependant, de la République aux Lois, qu'une attention accrue est portée aux liens qui unissent les auteurs de la reproduction. Dans la communauté idéale des femmes et des enfants, les mariages n'étaient en somme que des associations momentanées de couples anonymes grâce auxquelles les magistrats pouvaient, au prix de pieux mensonges, garantir une postérité de choix. C'était aussi, pour les gardiens, «un repos du guerrier" : aux plus braves d'entre eux, on octroyait des accouplements plus fréquents ! Dans le régime des Lois, le mariage redevient une institution

10 Voir aussi IX, 879c-d, où il est dit que, par égard pour les dieux de la procréation

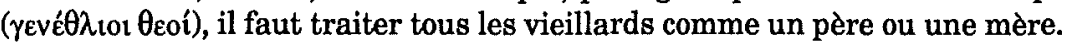


durable, et on a vu combien le législateur s'intéressait à la vie intime du foyer. Certes, la raison d'être principale du mariage que sanctifie la loi reste bien la procréation et l'éducation des enfants, mais on constate qu'ils n'en sont plus la justification exclusive. Ce qui, assez curieusement, le montre peut-être le mieux, c'est la situation d'exception que crée le divorce ${ }^{11}$. Le remariage étant permis, la préoccupation principale des magistrats doit être l'enfant pour ceux qui n'en ont pas eu ou pas assez, mais, dans le cas inverse, c'est le fait de "permettre à un homme et une femme de vieillir ensemble en prenant soin l'un de l'autre» (XI, 930a) ${ }^{12}$.

D'autres indications, relatives cette fois à la sexualité, témoignent d'un même souci de valorisation de la relation conjugale. A propos des passages de la République qui viennent d'être étudiés, on pouvait parler tout au plus d'une politique des relations sexuelles, centrée sur la procréation. Si la même préoccupation demeure dans les Lois, un point de vue éthique y apparait en rapport avec le couple et se traduit d'ailleurs par un renforcement des exigences conjugales. On peut dire, avec $\mathrm{A}$. Diès, que les règlementations en ce domaine ne visent pas seulement à «assurer la fécondité, mais, le mot n'est pas trop fort, la sainteté du mariage» ${ }^{13}$.

L'enquête sur les lois, est-il dit au début du dialogue (I, 636d), porte presque entièrement sur les plaisirs et les peines qui affectent la cité et l' $\hat{\eta} \theta 0 \varsigma$ des particuliers. Trouver en ce domaine la bonne mesure est la condition du bonheur ${ }^{14}$. Ces considérations viennent en conclusion d'un développement où Platon déplore la corruption des plaisirs de l'amour qu'ont encouragée certaines cités. Il faut considérer, dit-il, que le sexe féminin et le sexe masculin ont reçu ce plaisir de la nature afin de s'accoupler en vue de la génération. Les relations homosexuelles, qui sont $\pi \alpha \rho \grave{\alpha} \varphi v ́ \sigma \imath$, sont donc le fait d'une intempérance dans le plaisir.

Le thème de l'éros revient plus loin, juste avant que soit énoncée la loi sur la procréation. Des trois appétits fonciers qui nous habitent, il vient le plus tard, mais il est le plus vif, et c'est un feu plein de démesure. On doit donc le

11 Il n'est consenti que dans les cas d'incompatibilité de caractère, dûment vérifiée par les magistrats, et après des essais de conciliation (XI, 930a).

12 Peut-être est-il significatif que, pour ces «remariages», on ne voit pas apparaitre le

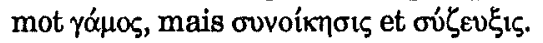

13 Cf. Platon, La République, Paris, Belles Lettres, t. 1, Intr., p. LXVIII.

14 Un long chapitre consacré à l'éthique personnelle (V,730c-734d) montre bien que Platon n'est nullement un adversaire du plaisir comme tel. Il est légitime, dit-il, de rechercher le plus de jouissance et le moins de peine tout au long de notre vie. Mais il n'est pas de plaisir authentique et durable sans une rectitude, qui est conformité à notre nature. Le débauché qui cède à la furie des amours n'est pas tel de son plein gré. Ce n'est pas cette vie que notre nature veut vraiment et qui est plaisante. 
contenir par la crainte, la loi, la raison droite, et en ayant recours, assurément ( $\mu \varepsilon ́ v \tau$ 'o), aux Muses ainsi qu'aux dieux qui président aux jeux (VI, 783a).

Ainsi la loi qui commande de procréer dans le mariage interdit-elle, tant pour l'homme que pour la femme, toute relation sexuelle extra-conjugale. Toutefois, en ce qui concerne les personnes qui ont passé l'âge de procréer, il ne faudrait réglementer semblablement que si le plus grand nombre ne faisait pas preuve de la modération voulue et amenait le désordre. Si ce n'est pas le cas, que l'honneur ou le déshonneur soit la sanction (VI, 784e). On remarque à nouveau que l'éthique des relations conjugales n'est pas fondée seulement sur des impératifs politiques relatifs à la procréation, mais sur les exigences d'une saine tempérance.

De la "victoire sur les plaisirs, condition du bonheur", il est encore question dans un long chapitre du livre VIII (835b-842a) où le philosophe entreprend une véritable éducation à l'amour, dans une perspective qui intéresse plus directement notre propos. L'exposé sert de préambule à une législation idéale sur les $\alpha \varphi \rho o ́ \delta ı \sigma \iota \alpha$ qu'il médite. Il est important, dès l'abord, souligne-t-il, de s'interroger sur la nature de l'éros afin de dissiper les équivoques qu'entretient ce mot. On distinguera donc trois formes de cet attachement ( $\varphi$ í $\lambda \circ v)$ très vif qu'est l'amour. Il y a l'amitié des semblables qui est douce et se caractérise par une réciprocité pouvant durer toute une vie;

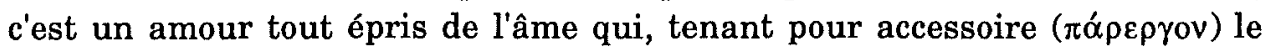
désir du corps, considérerait comme une injure le fait de vouloir se rassasier

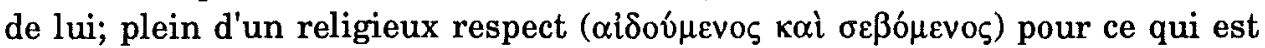

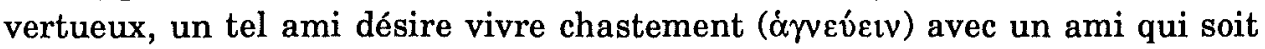
chaste lui aussi ${ }^{15}$. Le deuxième amour procède d'une amitié des contraires qui est redoutable, sauvage et rarement partagée; affamé qu'il est de la fleur de la jeunesse, cet amour n'hésite pas à se repaître des mœurs de l'aimé. Le troisième amour est un composé des deux premiers et se trouve donc tiraillé en sens contraires (837a-d).

15 Le mot $\sigma \varepsilon ́ \beta \varepsilon \sigma \theta \alpha \iota$ a toujours, chez Platon, une connotation religieuse; il s'emploie à propos des dieux, de la patrie ou de la famille. Les mots de la racine de $\alpha \gamma v o ́ \varsigma$ ont trait le plus souvent, dans la langue grecque, à la notion d'une pureté rituelle. Le domaine concerné est presque toujours celui de la génération et de la mort. Chez Platon, cette notion de pureté est intériorisée, moralisée et aussi spiritualisée : áyvós peut signifier la pureté transcendante qui caractérise le monde divin des Idées en ce qu'il est étranger au devenir, c'est-à-dire à la génération et à la mort. Voir notre étude citée à la note 4. Au sujet des règlements cultuels qui déterminent les règles de pureté rituelle, élément dont Michel Foucault ne tient pas compte, voir les remarques judicieuses de M. PIÉRART, art. cit. (note 1), p. 30-32. 
Le vocabulaire utilisé dans ce préambule montre bien que la relation à laquelle Platon se réfere pour établir cette distinction fondée sur des critères éthiques est celle de l'amour pour les garçons. Nul doute qu'il ne songe, comme modèle du premier éros, à l'affection qui unit le maître et le disciple et que vivifie une quête commune du vrai. Mais qu'en est-il des liens entre l'homme et la femme ? Ce n'est pas dit expressément. La radicalité du premier amour, - $\alpha \gamma v \varepsilon v ́ \varepsilon ı v$ suggère bien l'abstention de tout rapport charnel, - paraît bien en exclure la relation conjugale, puisque celle-ci a pour mission de procréer. Dans le principe cependant, rien n'empêche qu'elle puisse s'en rapprocher : elle peut tenir pour $\pi \alpha \dot{\alpha} \rho \varepsilon \rho \gamma o v$ le désir du corps et prétendre aussi à la réciprocité ainsi qu'à la durée, toutes qualités attribuées au premier amour. Si elle n'est pas comme telle orientée vers la quête du vrai, elle est du moins un lieu où peuvent se cultiver bien des vertus.

A la différence du préambule, la législation sur les $\alpha \varphi \rho o \delta i ́ \sigma \alpha \alpha$ nomme expressément les relations conjugales et nous ramène aussi à la dimension religieuse du sujet. Elle va tellement à contre-courant des mœurs reçues, note d'abord Platon, qu'elle ne pourrait s'imposer que grâce à ces trois rectitudes que sont la piété $(\theta \varepsilon \circ \sigma \varepsilon \beta \eta ́$ s), le sens de l'honneur et le désir des mœurs honorables de l'âme plutôt que celles du corps (841c). En somme, c'est de la divinité elle-même que devraient venir pareilles instructions, à sup-

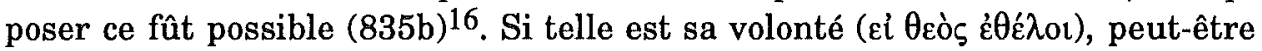
réussira-t-on néanmoins à l'imposer, fût-ce sous une forme atténuée (841c).

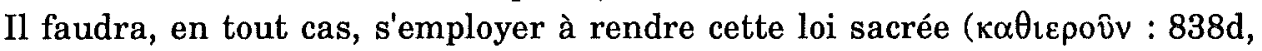
839c) afin que les actes qu'elle réprouve inspirent la même crainte religieuse ( $\mu \eta \delta \alpha \mu \hat{\omega} \varsigma$ ŏ $\sigma \iota, \theta \varepsilon o \mu \iota \sigma \hat{\eta}: 838 \mathrm{~b})$ que les relations incestueuses : celles-ci sont si unanimement réprouvées qu'elles ne suscitent même plus le désir chez le plus grand nombre.

La règle générale serait qu'il faut user conformément à la nature de l'accouplement destiné à la procréation ( $\tau \hat{\eta}_{\zeta} \pi \alpha \imath \delta o \gamma o v i ́ \alpha \varsigma$ $\sigma v v o v \sigma i ́ \alpha$ ), ce qui signifie l'abstention de toute relation homosexuelle ${ }^{17}$ et aussi de "tout champ féminin dans lequel on ne voudrait pas que croisse la semence déposée»

16 Les traditions religieuses de la Grèce gênent cependant Platon dans cet effort de

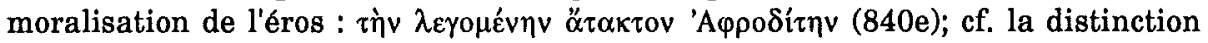
entre deux Aphrodite proposée dans le Banquet et l'article de V. PIRENNE-DELFORGE, Epithètes cultuelles et interprétation philosophique. A propos d'Aphrodite Ourania et Pandémos à Athenes, in AC, 57 (1988), p. 142-157.

17 La formulation mérite d'être soulignée : «qu'on ne touche pas au sexe mâle; qu'on ne tue pas délibérément la race humaine; qu'on ne jette pas la semence parmi les rocs et les cailloux où elle ne prendra jamais racine de façon à reproduire sa propre nature» (838e trad. des Places). Dans cette aspiration de l'homme à reproduire sa nature, on verra que Platon trouve un argument pour justifier le devoir de procréer. 
(839a). Dans un texte autrement formulé (841d), il est recommandé aux maris de n'avoir commerce ( $\sigma v \gamma \gamma \dot{i} v \varepsilon \sigma \theta \alpha \mathrm{l}$ ) qu'avec celles qui sont entrées dans leur maison avec les dieux et à la faveur de mariages sacrés ( $\mu \varepsilon \tau \grave{\alpha} \theta \varepsilon \hat{\omega} \mathrm{v}$

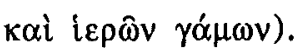

Si pareille législation ne pouvait s'imposer, les mœurs étant ce qu'elles sont, que la loi exige du moins de tenir cachées les pratiques contraires. Son respect ne serait pourtant pas au-dessus des forces humaines, ainsi que le montre l'exemple des athlètes et même de certains animaux : jusqu'à l'âge

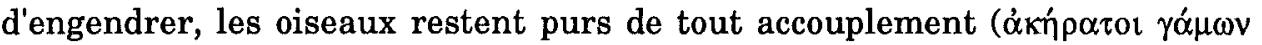
$\left.\tau \varepsilon \dot{\alpha} \gamma v i^{\prime}\right)$ et, quand le temps est venu, ils ne s'unissent qu'entre sexes

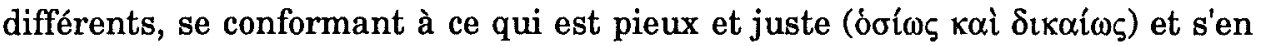
tenant à leurs premiers liens d'amitié ( $840 \mathrm{~d}-\mathrm{e})$. Et si cette loi pouvait être appliquée, elle serait à tous égards bénéfique, sa conformité à la nature étant son premier mérite. Elle détournerait notamment les hommes de la folie amoureuse, de l'adultère, des excès dans le boire et le manger, faisant en

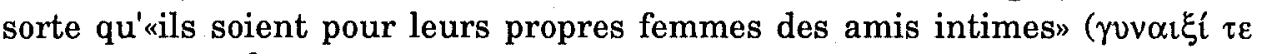

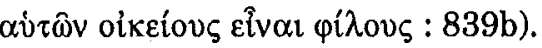

Ce long texte si dense, dont nous n'avons produit qu'un résumé succinct, mériterait certes une analyse très approfondie. Risquons néanmoins une première conclusion. Si la relation conjugale ne représente pas pour Platon la plus haute forme de l'amour humain, il la tient néanmoins en estime; il vise à la protéger, et il est très attentif à en souligner le caractère sacré. La valeur qui lui est reconnue tient certes à ce qu'elle permet la transmission de la vie, - c'est bien là sa finalité première, - mais au fait aussi qu'elle est une forme de $\varphi \imath \lambda i \alpha$ pouvant contribuer au progrès moral; en particulier, elle devient, aux yeux du philosophe, le seul lieu légitime où puisse s'apprendre le bon usage du plaisir sexuel ${ }^{18}$.

Sans doute faudra-t-il attendre le stoïcisme et Plutarque pour que se développe au sujet de l'éthique et de la spiritualité conjugales une réflexion philosophique dont on ne perçoit ici qu'une amorce timide. Prétendre cependant avec $M$. Foucault que «l'obligation de limiter les activités sexuelles au mariage concerne l'équilibre de la cité, sa moralité publique, les conditions d'une bonne procréation, et non les devoirs réciproques afférents à une

18 Il est un autre lieu où s'apprend le bon usage des plaisirs, concernant cette fois le boire, ce sont les chœurs et les fêtes de Dionysos. Platon recommande expressément de ne pas interdire les beuveries, mais de les réglementer de manière à ce qu'on s'y entraîne à la tempérance : Lois, II, 671a-672d. 
relation duelle entre les conjoints» 19 nous paraît relever d'une interprétation trop unilatérale des Lois.

\section{La justification métaphysique et religieuse de la procréation}

\section{Les Lois : participer à l'immortalité et servir la divinité}

Nous avons réservé jusqu'ici deux passages importants où Platon s'attache à dire le pourquoi de la procréation et à en fonder l'obligation. Le premier texte donne un exemple de préambule justificatif aux lois; celle qui est choisie et que le législateur détaillera plus loin concerne précisément l'obligation de se marier :

On se mariera de trente à trente-cinq ans dans la pensée qu'il est une manière dont la race humaine, par une disposition de sa nature, participe à l'immortalité ( $\dot{\alpha} \theta \alpha \vee \alpha \sigma i \alpha)$, chose dont tout homme éprouve aussi naturellement le désir sous tout rapport : devenir célèbre, en effet, et ne pas rester sans nom après qu'on soit mort est bien un désir d'une telle chose. La race humaine a donc une affinité naturelle ( $\sigma \cup \mu \varphi \cup n ́ s)$ avec la totalité du temps, et cette affinité sans cesse l'accompagne et l'accompagnera. La manière pour cette race d'être immortelle ( $\dot{\alpha} \theta \dot{\alpha} v \alpha \tau \sigma v)$, c'est de laisser des enfants de ses enfants ( $\tau \hat{\omega} \pi \alpha \hat{i} \delta \alpha \varsigma \pi \alpha i \delta \omega v$ $\kappa \alpha \tau \alpha \lambda \varepsilon \imath \pi$ ó $\mu \varepsilon v o v)$; elle est ainsi toujours la même et une, et par la généra-

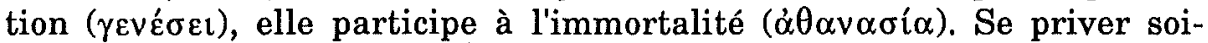

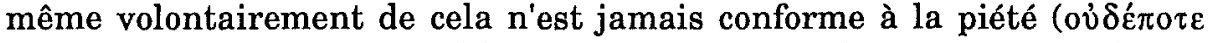
ö́tov) et c'est de propos délibéré que s'en prive celui qui néglige d'avoir enfants et femme (IV, 721b-c).

L'argumentation souligne tout d'abord l'aspiration naturelle de l'homme à l'immortalité. On notera ici le soin que met Platon à préciser que ce désir ne concerne pas seulement l'espèce humaine, mais chaque individu en particulier, et sous tout rapport; les exemples choisis, recherche de la gloire et survie du nom, sont bien propres à rendre chacun conscient de la présence en lui de cette aspiration fondamentale dont la génération est une autre expression.

De cette constatation le philosophe tire un impératif qu'il présente comme religieux (öбıov), mais il ne cherche pas ici à s'en expliquer. Sans doute

19 M. FouCAULT, L'usage des plaisirs, p. 188; il nous paraît aussi que l'auteur (p. 54) sous-estime limportance que Platon attache au critère de la nature pour apprécier les relations sexuelles. Le Politique (310 a-d) traite brièvement du mariage et de la procréation qui doivent préoccuper le «royal tisserand», mais aucune notation religieuse ne se rencontre dans ce passage. 
n'était-ce pas nécessaire, les Grecs étant spontanément portés à confondre l'ordre naturel et l'ordre divin. Au surplus, la référence à l'idée même d'immortalité suffisait à induire un impératif : comment refuser de suivre une aspiration qui permet aux hommes de partager un tant soit peu ce privilège par lequel les «Immortels" sont eux-mêmes ce qu'ils sont ${ }^{20}$ ?

Le second passage, inséré cette fois dans le préambule à la législation, reprend, sous une forme condensée et moins philosophique, l'argumentation du premier texte, auquel il se réfère d'ailleurs explicitement; il en précise ainsi la portée religieuse :

Il faut s'attacher à l'éternelle fécondité de la nature en laissant des enfants de ses enfants et en ne cessant ainsi de procurer à la divinité des serviteurs qui prennent notre place 21 .

S'inscrire dans ce perpétuel courant de la vie afin de maintenir le service du divin, faire en sorte que le devenir rejoigne l'immortel, tel est le sens de l'aspiration vitale qui habite l'homme, telle est sa vocation : il est un serviteur de la divinité 22 . Il existe donc une continuité profonde entre la nature universelle, l'humain et le divin. «Laisser des enfants de ses enfants» : la formule est traditionnelle et reprise deux fois. C'est par la procréation que se réalise, dans le devenir, cette permanence, et l'on a vu combien Platon, lorsqu'il parle de la famille, s'attache à faire sentir la continuité vraiment sacrée qui existe entre ses membres, vivants et morts, et qui les relie aux dieux. Toute l'argumentation qui vient d'être présentée

20 L'expression de cet impératif religieux trouve, chez Platon lui-même, d'autres formes. Le Timée (91a), par exemple, montre que ce sont les dieux qui ont formé en l'homme l'amour de la conjonction charnelle; le Phédon (61c-e) fait voir d'une autre manière que les dieux sont maitres de la vie : les hommes étant leur propriété, nul ne peut sans impiété ( $\theta \varepsilon ́ \mu 1 \tau o v)$ mettre fin à ses jours sans leur assentiment.

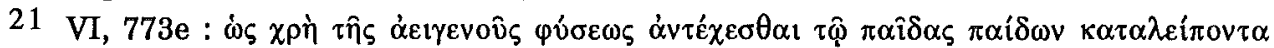

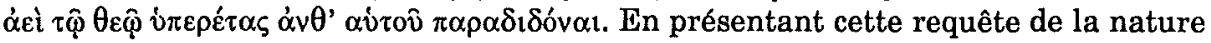
comme impérative, Platon prend l'exact contre-pied de la position de Démocrite (fr. 276-278 D.K.). Cf. notre étude : Le nécessaire, le naturel et l'agir humain selon Démocrite, in Proceedings of the Ist International Congress on Democritus, Xanthi, 1984, p. 339-345.

22 Cf. Phédon, 85a-b : Socrate est serviteur d'Apollon. Cette conception s'accorde bien avec la théologie que développent ailleurs les Lois : la divinité est origine et fin des existences; chercher, autant qu'il est possible, à lui ressembler et à en être l'ami, tel est le but que l'homme doit viser et d'où découle la plus belle des règles : «faire des sacrifices, entrer sans cesse en relation avec les dieux par des prières, des offrandes et tout l'ensemble du culte divin» (Lois, IV, 716a-717a). L'Epinomis (980b) dira : "passer sa vie à honorer les dieux en leur faisant hommage de ses hymnes et de son bonheur". 
n'est en somme qu'une expression philosophique, et donc universalisée, de cette prise de conscience existentielle qui s'éprouve tout d'abord au sein de la famille et qui doit s'élargir à la dimension de la cité et de l'univers tout entier ${ }^{23}$.

\section{Le Banquet : de la procréation physique à la procréation sprirituelle}

On a remarqué depuis longtemps ${ }^{24}$, touchant le thème de la procréation et de l'amour, une convergence fondamentale entre l'enseignement du Banquet et celui des Lois. Le premier développe, sous un mode spéculatif et en partie mystique, un message dont s'inspire le second dans l'optique politique et normative qui est la sienne. Le législateur athénien des Lois a bien dû rencontrer la prêtresse de Mantinée...

En son sens le plus général, explique Diotime, l'amour est le désir de possession perpétuelle de ce qui est bon; la manière particulière dont il se

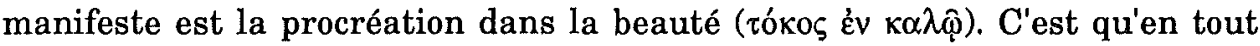
homme existe une fécondité (кuعî) et que sa nature, quand vient l'âge, aspire

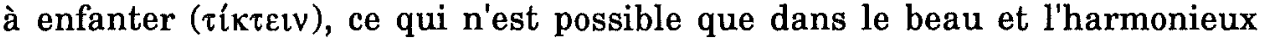

23 L'argument du service des dieux se rencontre, sous une forme inversée et plaisante, - les dieux ont besoin des hommes, - dans le mythe que le Banquet (190c) prête à Aristophane : anéantir la race humaine, pense Zeus, ce serait priver les dieux des honneurs et des sacrifices qu'ils reçoivent. L'idée affleure souvent dans les mythes, par exemple, dans celui que rapporte l'Hymne homérique à Déméter (310-313) : la sécheresse provoquée par la déesse menaçant d'anéantir la race humaine, Zeus prend conscience que les Olympiens risquent ainsi de se voir frustrés des offrandes et sacrifices, et il met tous ses soins à hâter un compromis entre les divinités en conflit. Mais il est d'autres rapprochements à faire entre ce poème métaphysique et la pensée religieuse de Platon et qui portent sur cette notion de solidarité et de continuité entre la nature, l'homme et le divin ainsi que sur l'aspiration humaine à l'immortalité. Déméter, qui incarne cette éternellle fécondité de la nature, est accueillie dans la famille royale d'Éleusis. Le vœu qu'elle adresse aux jeunes filles qui l'ont accueillie avec beaucoup de grâce est que ules dieux leur octroient de jeunes époux et leur donnent d'engendrer des enfants selon le désir de leurs parents" (135136). Elle-même souhaite devenir la nourrice d'un nouveau-né; elle va même entreprendre d'immortaliser le petit Démophon, mais ne pourra pas faire qu'il échappe à son destin de mort (260-261). Elle compensera cet échec par le don gratuit de ses mystères qui garantissent une vie et une survie bienheureuses, pourvu que les hommes l'honorent. Nous reviendrons ailleurs sur ce parallele qui introduit bien à la doctrine du Banquet, cette autre histoire d'amour.

24 Par exemple L. RoBIN, in Platon, Banquet, Paris, Belles Lettres, Intr., p. XLVI sq., et déjà dans son ouvrage plus ancien sur La théorie platonicienne de l'amour, Paris, 1908. 


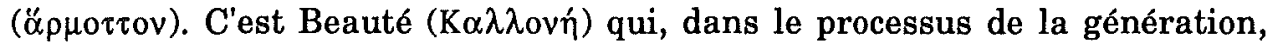
fait l'office d'Ilithye (206c-d).

Il faut bien voir que ce qui est impliqué par Platon dans cette idée de beauté, c'est notamment la présence d'un autre. L'enfantement ne peut se faire seul, il suppose un commerce, une relation harmonieuse avec autrui. Toutefois, relativement à l'objet du désir, cet autre n'apparaît que comme un intermédiaire, un accoucheur de ce dont on est gros soi-même. Du moins en va-t-il ainsi dans l'expérience humaine la plus courante. Ce que la nature mortelle, en définitive, cherche par l'enfantement, c'est à se perpétuer dans l'existence. Ainsi, comme va le préciser Diotime, l'amour a-t-il pour objet à la fois la procréation dans la beauté et l'immortalité (207a).

Or, il a été annoncé d'emblée (206c) que cette fécondité était double, s'exerçant selon le corps ou selon l'âme ${ }^{25}$. La prêtresse expose en premier lieu ce qui concerne l'activité somatique, et voici ce qu'elle en dit :

L'union (ovvovoía) de l'homme et de la femme est en effet un enfantement ( immortel chez ce vivant mortel, c'est la fécondité et la procréation 26 .

L'union du couple exprime donc l'aspiration de l'homme à l'immortalité et cela lui confère une dimension religieuse. Le passage parallèle des Lois exprimait en d'autres termes la même idée : la race humaine participe à l'immortalité par la génération, et il serait impie de se priver de ce privilège divin.

Comme autre signe de la présence en l'homme d'une aspiration à l'immortalité, le Banquet (208c) évoque pareillement son «amoureux désir de se faire un nom et de s'assurer pour l'éternité une gloire impérissable». Plus abondante encore que celle du corps, la fécondité selon l'âme concerne la pensée et toute espèce d'excellence $(209 a)$. Un processus semblable à celui de la procréation physique se produit chez celui qui, dès son jeune âge, porte en lui cette fécondité. C'est à force de fréquenter une âme belle et noble, quand la tendresse ( $\dot{\alpha} \sigma \pi \alpha \dot{\alpha} \zeta \varepsilon \sigma \theta \alpha \mathrm{l})$ est à son comble, qu'il se met à enfanter et à éduquer; de nourrir ces enfants, plus beaux et plus immortels que les enfants

25 Il s'ensuit que tout le vocabulaire qu'utilise Platon s'applique aux deux domaines, ce qui ne va pas sans équivoque. Il y aurait intérêt à examiner de très près cette terminologie, mais on pressent que, pour Platon, la métaphore exprime une réalité profonde. On sait les variations du Théétète sur la maieutique.

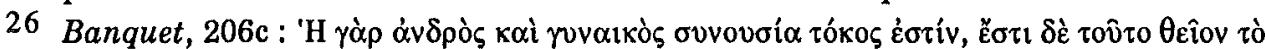

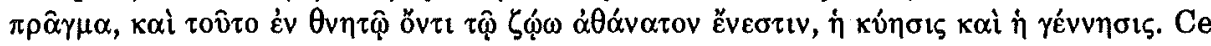
texte qui paraît interrompre le développement a souvent été considéré comme une glose; on ne pourrait cependant le supprimer sans modifier aussi ce qui suit. 
de chair, il se crée entre les deux amis une communauté et une affection $\left(\varphi \imath \lambda i^{\alpha} \alpha\right)$ incomparables $(209 \mathrm{c})$.

Dans la manière, quelle qu'elle soit, dont l'être mortel s'efforce ainsi d'assurer «une repousse de lui-même», gît toujours, cependant, un artifice : l'être engendré n'est jamais qu'un être nouveau, distinct de celui qui engendre, alors que l'existence divine est quant à elle toujours identique à elle-même $(208 b)^{27}$. C'est d'une autre manière qu'elle est immortelle.

C'est bien à cette immortalité-ci pourtant que l'homme aspire. Mais pour s'en approcher, il lui faut l'initiation parfaite, l'époptie. Parvenu à ce sommet, ce n'est plus à un être beau particulier que l'on s'unit, mais c'est la Beauté elle-même, merveilleusement, éternellement, universellement belle, que l'on découvre tout d'un coup et que l'on contemple. Et l'union ( $\xi \hat{v} \omega \omega \mathrm{v})$ à

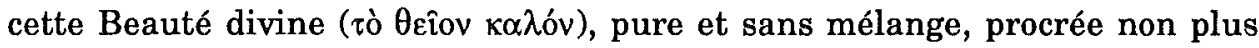
des images d'excellence, mais des excellences véritables. C'est à celui qui engendre ce fruit réel que revient le titre d'ami des dieux ( $\left(\varepsilon_{\varepsilon} \varphi \iota_{\imath} \lambda \eta_{\zeta}\right)$ et c'est lui aussi qui peut être immortel ( $\dot{\alpha} \theta \alpha \dot{\alpha} v \alpha \tau \varsigma)$ ), si tant est qu'un homme peut l'être (210e-212a).

\section{Conclusion}

Vue de ce sommet où s'est hissée Diotime, la procréation que réalise l'union de l'homme et de la femme ne peut qu'apparaître dévalorisée ${ }^{28}$. La

27 C'est évident dans le cas de la fécondité physique et ce l'est aussi pour la fécondité dite psychique quand on prend pour exemples d'«immortels enfantements" les poèmes d'Homère et d'Hésiode ou la législation de Lycurgue et de Solon qui leur ont valu bien souvent des cultes (iepó) (209c). D'autre part, on comprend mieux par la physiologie de l'acte sexuel qu'expose le Timée (91a-c) ce qu'évoque la perte (ó $\jmath_{0} \lambda \lambda v \mu \mathrm{l}$ ) dont parle le Banquet (207d) à propos des perpétuels renouvellements qui s'opèrent en l'homme, car c'est bien ce qui caractérise aussi la génération : le sperme, qui est une partie de la moelle et est en liaison avec le cerveau, est un vivant doué d'une âme, aussi bien chez la femme que chez l'homme, et l'ouverture par lequel il respire lui donne le désir de s'écouler au dehors. L'homme se prive donc, dans l'acte sexuel, d'une substance vitale. On peut dire qu'il fait par là l'expérience à la fois de la mort et de l'immortalité. M. Foucault (op. cit., p. 150-156) montre cela fort bien à propos de Platon et d'autres penseurs antiques. On notera aussi que le cerveaumoelle est comparé à une glèbe qui reçoit le "sperme divin" qu'est l'intellect (73c); l'union sexuelle est en somme une imitation de cette fécondation supérieure. Le Timée $(90 \mathrm{~b})$ brode aussi sur le thème de la participation à l'immortalité, mais uniquement psychique.

28 Qui touche à l'immortalité véritable peut se dispenser des participations inférieures. C'est le lieu de rappeler que Platon était célibataire. Dans la tradition chrétienne, il est bien connu qu'au ciel la question du mariage ne se pose plus, parce qu'on n'y meurt pas, comme le précise le Christ dans sa réponse aux Sadducéens $(L c, 20,36)$. Sur la 
dévalorisation n'est toutefois que relative, car cette union réalise bien à sa manière une participation de l'homme à l'immortalité; elle est à ce titre une imitation du divin et, pour peu qu'elle sache se soustraire aux déviations passionnelles de l'éros, rien n'exclut qu'elle soit un jalon vers des noces spirituelles plus fécondes. Il ne semble pas que, dans le Banquet, Platon ait voulu désavouer cet amour humain, mais le point de vue plus «terrestre» qui est le sien dans les Lois l'amène manifestement à en souligner davantage certains éléments positifs.

Considérée rétrospectivement, à la lumière de l'enseignement de ces deux dialogues, la République fait songer, concernant le thème de la procréation et sa dimension sacrée, à l'exercice de style d'un poète-logicien façonnant une constitution idéale. Son discours religieux évoque ce législateur habile que mettait en scène le sophiste Critias : observant que la sanction des lois ne suffisait pas à détourner les hommes des méfaits commis en cachette, il avait eu l'idée de leur inspirer la crainte d'un être divin dont la redoutable puissance s'alliait à une sagesse tenant toutes choses sous son regard 29 !

Or le Banquet, œuvre de la maturité, et les Lois, œuvre de l'extrême vieillesse, montrent bien que telle n'a jamais été la véritable pensée politicoreligieuse de Platon. Certes, il reste à tout moment persuadé que la religion est indispensable au bon gouvernement de la cité et qu'elle est, pour chaque citoyen, la meilleure sauvegarde d'une bonne conduite, mais il proclame aussi que «la vérité vient en tête de tous les biens» (Lois, V, 730c). S'il importe donc d'accréditer l'idée que la procréation humaine est chose sacrée, c'est parce qu'elle l'est véritablement. Telle est la conclusion qui ressort du Banquet et du préambule des Lois. L'homme procrée parce qu'il est mortel, mais en procréant il se met en chemin vers l'immortalité.

Université de Liège

André MOTTE

Place du XX-Août, 32

B - 4000 LIÈGE.

différences entre les types d'immortalité, voir J. BELs, Athanasia ou la fausse immortalité, in RHR, 202 (1985), p. 115-124.

29 Le fragment est repris dans DIELS-KRANZ, FVS, 88 A 25. 